Pacific Journal of Mathematics

COMBINATORIAL AND GEOMETRIC PROPERTIES OF

作 


\section{COMBINATORIAL AND GEOMETRIC PROPERTIES OF WEIGHT SYSTEMS OF IRREDUCIBLE FINITE- DIMENSIONAL REPRENSENTATIONS OF SIMPLE SPLIT LIE ALGEBRAS OVER FIELDS OF 0 CHARACTERISTIC}

\section{BENEDICT SEIFERT}

Let $R$ be a simple split Lie algebra over $K$, a field of 0 characteristic. Let $\pi=\pi\left(\lambda^{+}\right)$be the representation with highest weight $\lambda^{+}$. Let $W t\left(\lambda^{+}\right)$be its weight system. Let $S$ be a subset of the root system. We define a graph $g r_{S}\left(\lambda^{+}\right)$ whose set of nodes is $W t\left(\lambda^{+}\right)$and set of links is given by pairs of weights whose difference is a root in $S$. In particular taking $S=\Sigma^{0}$, the system of simple roots, we investigate the properties of representations $\pi\left(\lambda^{+}\right)$such that gr ${ }^{0}\left(\lambda^{+}\right)$is simply connected. We give a complete list of these, for each simple Lie algebra.

We then attach to $\pi\left(\lambda^{+}\right)$an affine rational lattice $L\left(\lambda^{+}\right)$, the root of $\lambda^{+}$in the weight lattice modulo the root lattice and a rational polyhedron, $C\left(\lambda^{+}\right)$, the rational convex closure of the Weyl group orbit of $\lambda^{+}$We. give the following geometric characterization of the weight system: $W t\left(\lambda^{+}\right)=$ $L\left(\lambda^{+}\right) \cap C\left(\lambda^{+}\right) .^{1}$

o. Preliminaries. 1. We shall consider a simple Lie algebra $R$ split over a field of characteristic $0, K$, of rank $r$. For any vector space $V, V_{K}^{\prime}$ will denote the $K$-dual of that space, or $V^{\prime}$, if $K$ is understood. We denote a Cartan subalgebra by $\sqrt{ }$, the system of roots, positive roots, and simple roots, respectively by $\Sigma, \Sigma^{+}$, and $\Sigma^{0}$. We denote the $Q$-space spanned by $\Sigma$ by $\sqrt{ }^{0}$. The Killing form on $R$ defines inner products on the spaces $R, R_{K}^{\prime}, \sqrt{ }, \sqrt{ }^{0}, \sqrt{ }_{K}^{\prime}, \sqrt{ }_{Q^{\prime}}^{0}$ in the usual manner; we denote any of these by (, ). We denote the co-roots corresponding to $a \in \Sigma$ by $H_{a} . \quad H_{a}$ is the element of $\sqrt{ }$ defined by the conditions $b\left(H_{a}\right)=2(a, b) /(a, a)$ for $b \in \Sigma$. The set of co-roots will be denoted by $\Sigma^{*}$. The system of fundamental weights will be denoted by $F^{0}=\left\{\lambda_{a}\right\}_{a \in \Sigma^{0}}$. It is the dual basis to $\Sigma^{*}$ in $\sqrt{ }^{\prime}$. Given $a \in \Sigma$ we define $t_{a}$ to be the 'root subalgebra' or R.S.A., i.e., the natural copy of $\mathrm{sl}(2)$ imbedded in $R$ spanned by the $a,-a$ not spaces and $H_{a}$. We fix in each such root subalgebra a basis such that the commutation relations are given by

$$
\left[H_{a}, X_{a}\right]=2 \cdot X_{a}
$$

1 Added in proof. For a result equivalent to 2.10. See F. Berezin and I. Gelfand, Trudy Hoshov, Mat. Obščesvo. 6 (1957), 371-463; the proof there is analytic. 


$$
\begin{aligned}
& {\left[H_{a}, X_{-a}\right]=-2 \cdot X_{-a}} \\
& {\left[X_{a} \cdot X_{-a}\right]=-H_{a} .}
\end{aligned}
$$

In addition we can make such a choice in such a way that the commutation relations in $R$ are given-in addition to (0.1)-by (0.2), for $a \neq-b, a, b, a+b \in \Sigma$ :

$$
\left[X_{a}, X_{b}\right]= \pm f(a, b) X_{a+b}
$$

with $f(a, b)$ the smallest integer $n \geqq 0$ such that $b-f(a, b) \cdot a \notin \Sigma$; the sign in (0.2), which can be computed in terms of certain special Chevalley bases (see [2]) doesn't concern us.

$L$ and $L(\Sigma)$ will denote, respectively, the weight and root lattice of $R$, the positive cones in these lattices with respect to the bases $F^{0}$ and $\Sigma^{0}$, respectively, by $L^{+}$and $L^{+}(\Sigma)$. Given an element of $L^{+}, \lambda^{+}$, we denote the unique finite-dimensional irreducible representation with highest weight $\lambda^{+}$, by $\pi\left(\lambda^{+}\right)$. The weight system of $\pi\left(\lambda^{+}\right)$will be written $W t\left(\lambda^{+}\right)$. When $R=$ sl(2), any such representation $\pi=\pi\left(\lambda^{+}\right)$can be realized in a certain normal form introduced in [2] which we shall call Tits normal form. With respect to a basis in $\mathrm{sl}(2)$ as in (0.1) this normal form is as follows

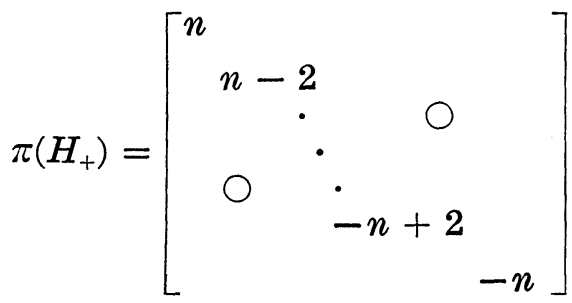

$$
\begin{aligned}
& \pi\left(X_{+}\right)=\left[\begin{array}{cccc}
0 & 1 & & \\
& 0 & 2 & 0 \\
& \ddots & \ddots & \\
& \ddots & \ddots & \\
& & & 0 \\
& & & 0
\end{array}\right] \\
& \pi\left(X_{-}\right)=\stackrel{v_{n}}{\cdot} \cdot\left[\begin{array}{ccccc}
0 & & & v_{-n} \\
-n & 0 & 0 & \\
-n+1 & 0 \\
& \cdot \\
v_{-n} & 0 & \ddots & \\
& & \ddots & 0 \\
& & & -1 & 0
\end{array}\right] .
\end{aligned}
$$

Given a finite-dimensional representation $\pi$ of $R, \pi_{a}$ will denote the restriction of $\pi$ to $t_{a}$. 
2. Given a set $S$ and a relation $R \subseteq S \times S$, we let $R^{\prime}$ be the transitive closure of $R$, i.e., $R^{\prime}$ consists of all pairs $\left(x, x^{0}\right)$ such that there exists a sequence $x=x_{1}, \cdots, x_{n}=x^{0}$ with $\left(x_{i}, x_{i+1}\right) \in R$, for $i=1, \cdots, n-1$. We say that $R$ is anti-symmetric if $(x, y) \in R$ implies $(y, x) \notin R . \quad R$ is said to be strongly anti-symmetric iff $R^{\prime}$ is anti-symmetric. Then we make the following basic

Definition 0.3. A directed graph (or simply graph) is a pair $g r=(N(g r), L k(g r))$ with $N(g r)$, a set, the set of 'nodes', and $L k(g r)$ a strongly anti-symmetric relation on $N(g r)$, the set of 'links'.

When $g r$ is understood we write $x<y$ for $(x, y) \in(L k(g r))^{\prime}$, and say that $y$ is above $x$ in $g r$. We abbreviate ' $x<y$ or $x=y$ ' by ' $x<=y$ '. $N(g r)$ is a partially ordered set with respect to $<$.

Given a graph $g r$ we define the opposite graph $g r^{0}$ by

$$
\begin{aligned}
& N\left(g r^{0}\right)=N(g r) \\
& L k\left(g r^{0}\right)=\{(x, y) \mid(y, x) \in L k(g r)\} .
\end{aligned}
$$

$x \in N(g r)$ is said to be an ascending branch point iff for some $y \neq y^{\prime},(x, y),\left(x, y^{\prime}\right) \in L k(g r)$. It is said to be a descending branch point if it is an ascending branch point of $g r^{0}$. A sequence $x_{1}, \cdots$, $x_{i}, \cdots$ such that $\left(x_{j}, x_{j+1}\right) \in L k(g r)$ is called a chain of $g r$. A loop in $g r$ is a pair of unequal chains whose first and last elements coincide. If there are no loops in $g r$, we say that $g r$ is simply connected.

A homomorphism of graphs is a set map from $N(g r)$ to $N\left(g r^{\prime}\right)$, $T$, which induces a map from $L k(g r)$ to $L k\left(g r^{\prime}\right)$ by the formula

$$
(x, y) \longrightarrow(T(x), T(y)) \text {. }
$$

Clearly every homomorphism of graphs from $g r$ to $g r^{\prime}$ induces a homomorphism of partially ordered sets from $(N(g r),<)$ to $\left(N\left(g r^{\prime}\right)\right.$, $\left.<^{\prime}\right)$. A homomorphism from $g r$ to its opposite graph $g r^{0}$ is said to be an anti-homomorphism. $g r$ is said to be involutive iff there exists an anti-automorphism of it, i.e., an anti-isomorphism from $g r$ to itself. If this anti-automorphism can be chosen to be of order 2 , then $g r$ is said to be symmetric.

We say that $g r$ is upper complete iff every proper subset of $N(g r)$ has an upper bound in $g r$, with respect to the partial ordering < defined by $g r$. (We don't require least upper bounds to exist.) If $g r^{0}$ is upper complete then $g r$ is said to be lower complete. Finally we say that $g r$ is Noetherian if the corresponding partially ordered set is, i.e., if all chains $x_{1}<x_{2}<\cdots$ are finite, and that $g r$ is downwards Noetherian if $g r^{0}$ is Noetherian.

Lemma 0.5. An upper complete Noetherian graph has a unique 
element $n^{+}$such that every element $n \neq n^{+}$in $N$ satisfies $n<n^{+}$. If $g r$ is involutive then there exists also a lowest element $n^{-}$such that $n^{-}<n$ for $n \neq n^{-}$.

Proof. Assume that no $n^{+}$satisfying the assertion exists. Then we can find an infinite ascending chain violating the Noetherian property and thus arriving at a contradiction in the following manner. Choose $n=n_{0}$ arbitrarily. Having chosen $n_{0}, \cdots, n_{i}$, we can choose $n_{i+1}>n_{i}$. For if this were not possible, i.e., if $n_{i} \nless n$ for all $n \in N$, then for any $m \in N$ we can apply the upper completeness property to the set $S_{m}=\left\{n_{i}, m\right\}$; we conclude that there exists $m^{\prime}$ such that $n_{i} \leqq m^{\prime}$, and $m \leqq m^{\prime}$. Now since $n_{i} \nless m^{\prime}$, we must have $n_{i}=m^{\prime}$. But then for each set $S_{m}=\left\{n_{i}, m\right\}, n_{i}$ is an upper bound for $S$. Hence $m \leqq n_{i}$ and $n_{i}=n^{+}$. This contradiction proves the existence of $n^{+}$. The uniqueness is immediate from the antisymmetry of $<$. Lastly if $N$ is involutive, then an involution takes $n^{+}$to $n^{-}$as defined.

Henceforth we shall say 'complete' for upper complete (which implies lower complete) when we are dealing with involutive graphs, and use the notation $n^{+}$and $n^{-}$as in the lemma.

Lemma 0.6. If $g r$ is a complete Noetherian involutive graph then T.F.A.E.:

(i) $g r$ has no ascending branch point.

(ii) gr has no descending branch point.

(iii) $g r$ is simply connected.

Proof. Any anti-automorphism takes an upper branch point to a lower branch point. Hence for involutive graphs (i) is equivalent to (ii). Obviously (iii) follows from (i) or (ii). To show that (iii) implies (i) assume that $n \in N(g r)$ is an ascending branch point. Consider the distinct elements of $L k(g r),\left(n, n^{\prime}\right),\left(n, n^{\prime \prime}\right)$. By the preceding Lemma 0.5 , we have $n^{\prime}, n^{\prime \prime} \leqq n^{+}$. By the definition of the relation $\leqq$ in $g r$, there exist chains in $g r, n^{\prime}=n_{1}^{\prime}, \cdots, n_{r}^{\prime}=n^{+}$and $n^{\prime \prime}=n_{1}^{\prime \prime}, \cdots, n_{s}^{\prime \prime}=n^{+}$. Appending $n=n_{0}$ to each of these ascending chains we obtain a loop. Hence $g r$ is not simply connected.

1. Graphs attached to irreducible representations. Now suppose $S \subseteq \Sigma$, and $\pi$ an irreducible representation of $R$ with highest weight $\lambda^{+}$and weight system $W t\left(\lambda^{+}\right)$. We attach to $\pi$ and $S$ the following graph denoted by $g r_{S}\left(\lambda^{+}\right)$:

$$
N\left(g r_{S}(\pi)\right)=W t\left(\lambda^{+}\right)
$$




$$
\operatorname{Lk}\left(g r_{S}(\pi)\right)=\{(\lambda, \lambda+a)\}_{\lambda \in W t(\lambda+), a \in S} \cdot
$$

We denote the graph $g r_{\Sigma^{0}}\left(\lambda^{+}\right)$by $g r\left(\lambda^{+}\right)$, and $g r_{\Sigma}\left(\lambda^{+}\right)$by $\operatorname{Gr}\left(\lambda^{+}\right)$. $\operatorname{gr}\left(\lambda^{+}\right)$is called the weight graph of $\pi\left(\lambda^{+}\right)$.

LEMMA 1.3. $\operatorname{gr}\left(\lambda^{+}\right)$is a directed finite complete Noetherian symmetric graph.

Proof. The finiteness being known, $g r(\lambda)$ is Noetherian. The completeness property is a restatement of the existence of a chain $\lambda^{+}=\lambda_{0}, \cdots, \lambda_{n}=\lambda$ with $\lambda_{i}-\lambda_{i+1}$ in $\Sigma^{0}$. We must show that the graph is symmetric.

Now, since the Weyl group acts simply transitively on the set of Weyl chambers there exists a unique element $w^{-} \in W$ taking the dominant, or positive, Weyl chamber (in terms of the ordering chosen), $\underline{C}^{+}$to the negative chamber $\underline{C}^{-}$. This element takes the positive roots to negative roots, and in particular $w^{-}$takes $\Sigma^{0}$ to $-\Sigma^{0}$. Now define the involution $w^{-}$of $g r\left(\lambda^{+}\right)$by $w^{-} \in W$ on the set of nodes of $\operatorname{gr}\left(\lambda^{+}\right), W t\left(\lambda^{+}\right)$. Then the set of links $\{(\lambda, \lambda+a)\}_{\lambda \in W t, a \in \Sigma^{0}}$ is taken to $\left\{\left(w^{-}(\lambda), w^{-}(\lambda)+w^{-}(a)\right\}\right.$ which, since $w^{-}(a) \in-\Sigma^{0}$, consists of elements in $\operatorname{Lk}\left(g r^{0}\right)$.

Since $\left(w^{-}\right)^{2}$ takes $\underline{C}^{+}$, the dominant chamber, to itself, it is the identity, by simple transitivity; so $w^{-}$is an anti-automorphism of order 2. Hence $\operatorname{gr}\left(\lambda^{+}\right)$is symmetric. We shall denote $w^{-}\left(\lambda^{+}\right)$by $\lambda^{-}$.

Given $a \in \Sigma^{0}$ we call a chain $\cdots, \lambda_{i}, \lambda_{i+1}, \cdots,\left(\lambda_{i}, \lambda_{i+1}\right) \in L k(g r)$ in $\operatorname{gr}\left(\lambda^{+}\right)$an $a$-chain if all links of the chain consist of pairs $(\lambda, \lambda+a)$. A maximal $a$-chain is an $a$-chain not properly contained in any other $a$-chain. We call $\lambda a$-extremal iff $\lambda$ is either the first or last element of any $a$-chain in $g r\left(\lambda^{+}\right)$of which it is a member. It is called $a$-maximal or minimal depending on which of these possibilities is realized. For $a \in \Sigma$, we have the analogous notion with respect to $\operatorname{Gr}\left(\lambda^{+}\right)$.

We say that $\lambda$ is $\Sigma^{0}$-extremal iff $\lambda$ is $a$-extremal for every $a \epsilon$ $\Sigma^{0}$. We say that it is extremal iff it is $a$-extremal for every $a \in \Sigma$ (i.e., extremal in $\operatorname{Gr}\left(\lambda^{+}\right)$, in the obvious sense).

LEMMA 1.4. $\lambda$ is extremal iff it is $\Sigma^{0}$-extremal.

Proof. $W$ acts on $\operatorname{Gr}\left(\lambda^{+}\right) . \quad w \in W$ takes $a$-strings to $w(a)$-strings; it takes $a$-extremal elements to $w(a)$-extremal elements. Hence since $\Sigma^{0}$ is a cross section for the action of $W$ on $\Sigma$ the two definitions are equivalent, since $\lambda^{+}$is extremal and the only $g r\left(\lambda^{+}\right)$-extremal weight in $\mathrm{C}^{+}$. 
We denote the set of extremal weights by $W t^{<}\left(\lambda^{+}\right)$. We can characterize this set in another way:

Proposition 1.5. $W t^{<}\left(\lambda^{+}\right)=W \cdot \lambda^{+}$, the Weyl group orbit of the highest weight.

Proof. It is obvious that $W \cdot \lambda^{+} \subseteq W t^{<}\left(\lambda^{+}\right)$, since, as in the proof of the preceding lemma, $W t^{<}\left(\lambda^{+}\right)$is invariant under $W$, and obviously $\lambda^{+}$is extremal, in fact $a$-maximal for all $a \in \Sigma^{0}$. We show the opposite inclusion by induction on the following function defined on $W t\left(\lambda^{+}\right)$: if $\lambda=\lambda^{+}-\sum_{\Sigma^{0}} n_{a} \cdot a$ then

$$
h(\lambda)=\sum_{\Sigma 0} n_{a} .
$$

So let $\lambda \in W t^{<}\left(\lambda^{+}\right)$be minimal with respect to $h$, such that $\lambda \notin W \cdot \lambda^{+}$. Since $\lambda \neq \lambda^{+}$and $\lambda^{+}$is the only weight which is $a$-maximal for all $a \in \Sigma^{0}$, there exists $a \in \Sigma^{0}$ such that $\lambda$ is $a$-minimal. Hence $\lambda\left(H_{a}\right)<$ 0 . Hence $h\left(w_{a}(\lambda)\right)=h(\lambda)+\lambda\left(H_{a}\right)<h(\lambda)$. By the $h$-minimality of $\lambda$ this means that $w_{a}(\lambda) \in W \cdot \lambda^{+}$. Therefore, $\lambda=w_{a}\left(w_{a}(\lambda)\right) \in W \cdot \lambda^{+}$. This contradiction proves the proposition.

We now make the following

DEFINITION 1.6. A representation $\pi=\pi\left(\lambda^{+}\right)$is said to be simply connected (or s.c.) iff the weight diagram $\operatorname{gr}\left(\lambda^{+}\right)$is simply connected.

We can enumerate all s.c. representations of each isomorphism type of simple Lie algebra.

THEOREM 1.7. Let $R$ be a simple Lie algebra of rank $>1$, and $\pi$ an s.c. irreducible representation with highest weight $\lambda^{+}$. Then $R$ is one of the following Lie algebras: $A_{p}, B_{p}, C_{p}$, or $G_{2}(p=1,2, \cdots)$ and $\lambda^{+}$is the fundamental weight corresponding to the root circled in the list of Dynkin diagrams below:

$A_{p}$
$B_{2}=C_{2}$
$C_{p>2}$
$B_{p>2}$
$G_{2}$

(0) 0
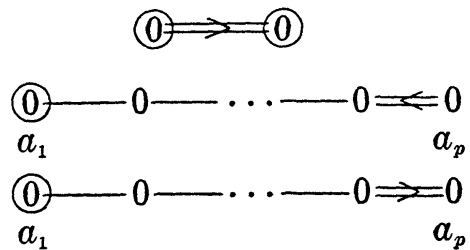

00
$b_{1}$

REMARKS. Notice that the theorem implies the following facts: 
(1) If $R$ is a simple split algebra with a Dynkin diagram that contains branch points $\left(E_{8}, E_{7}, E_{8}, D_{p}\right)$ then $R$ has no s.c. representations.

(2) If $\lambda^{+}$is the highest weight of an s.c. representation $\pi\left(\lambda^{+}\right)$ then $\lambda^{+}$is the fundamental weight corresponding to a node on one of the edges of the Dynkin diagram, i.e., is very singular with respect to the Weyl group action.

We now proceed to the proof of the theorem. $\pi$ will denote an s.c. representation.

Lemma 1.8. Suppose $\pi$ is an irreducible representation of $R$ and $\lambda \in W t\left(\lambda^{+}\right), a \in \Sigma^{0}, \lambda\left(H_{a}\right)=n>0$. Then $\lambda, \cdots, \lambda-n \cdot a$ is an a-chain passing through $\lambda$. $\lambda-n \cdot a=w_{a}(\lambda)$. The chain through $\lambda$ is maximal iff for $k>0, \lambda+k a$ (in case $n>0$ ) and $\lambda-k a$ (in case $n<0$ ) are not in the weight system $W t(\pi)$.

This lemma is standard theory.

LEMMA 1.9. If $\pi$ is simply connected, $\lambda^{+}$its highest weight, then there exists $a \in \Sigma^{0}$ such that $W_{a}^{\prime}$, the group generated by the reflections $\left\{w_{b}\right\}_{b \in \Sigma^{0}, b \neq a}$ is contained in the stabilizer of $\lambda^{+}$in $W$.

Proof. Suppose otherwise. Then there exists $a, a^{\prime} \in \Sigma^{0}$ such that $w_{a}\left(\lambda^{+}\right)=\lambda^{+}-\lambda^{+}\left(H_{a}\right) \cdot a$ and $w_{a^{\prime}}\left(\lambda^{+}\right)=\lambda^{+}-\lambda^{+}\left(H_{a^{\prime}}\right) \cdot a^{\prime}$ both are not equal to $\lambda^{+}$, i.e., $\lambda^{+}\left(H_{a}\right), \lambda^{+}\left(H_{a^{\prime}}\right)$ are two strictly positive integers (they are always nonnegative). So there are nontrivial $a$ - and $a^{\prime}$ strings passing through $\lambda^{+}, \lambda^{+}$being their maximal element. Hence $\lambda^{+}$is a descending branch point of $\operatorname{gr}\left(\lambda^{+}\right)$. Since $g r\left(\lambda^{+}\right)$is symmetric and complete (1.3), the existence of a branch point implies that $\operatorname{gr}\left(\lambda^{+}\right)$ is not simply connected (Lemma 0.6).

LEMma 1.10. For $\pi=\pi\left(\lambda^{+}\right)$s.c., $\lambda \in W t\left(\lambda^{+}\right)=N\left(\operatorname{gr}\left(\lambda^{+}\right)\right)$, there exist two perhaps distinct elements $a, a^{\prime} \in \Sigma^{0}$ such that $W_{a, a^{\prime}}^{\prime}$, the group generated by all reflections in simple roots distinct from a and $a^{\prime}$, is contained in the stabilizer of $\lambda$.

Proof. If the lemma were false there would, by the same computation as in the previous lemma, have to exist in $\operatorname{gr}\left(\lambda^{+}\right) 3$ nontrivial strings corresponding to distinct simple roots passing through $\lambda$. So there would have to be two distinct links either of the kind $(\lambda, \lambda+a) \in \operatorname{Lk}\left(g r\left(\lambda^{+}\right)\right)$or $(\lambda-a, \lambda) \in \operatorname{Lk}\left(g r\left(\lambda^{+}\right)\right)$, i.e., $\lambda$ would be either an ascending or a descending branch point of the weight diagram. This, again by Lemmas 1.3 and 0.6 , would imply that $g r\left(\lambda^{+}\right)$is not simply connected. 
Definition 1.11. A simple root of $R$ is called 'on edge' iff $a\left(H_{b}\right) \neq 0$ for exactly one $b \neq a$ in $\Sigma^{0}$.

The definition is motivated, obviously, by the Dynkin diagram.

Proposition 1.12. If $\pi$ is s.c. then its highest weight $\lambda^{+}$must be a fundamental weight $\lambda_{a}$ for some simple root a on edge.

Proof. Lemma 1.9 is equivalent to the assertion that $\lambda^{+}$is perpendicular to all but one simple root. Hence by definition $\lambda^{+}$ must be some multiple of some fundamental weight, say, $\lambda^{+}=n \cdot \lambda_{a}$. We want to show that $a$ is on edge. If not, there exist at least two roots in $\Sigma^{0}, b$ and $b^{\prime}$, say, such that $(a, b),\left(a, b^{\prime}\right)$ are smaller than zero. Then the weight $\lambda^{+}-a$ (by Lemma 1.8 it is a weight) satisfies

$$
\left(\lambda^{+}-a\right)\left(H_{b}\right)>0, \quad\left(\lambda^{+}-a\right)\left(H_{b^{\prime}}\right)>0
$$

and $\lambda^{+}-a$, again by Lemma 1.8, is a downwards branch point of $\operatorname{gr}\left(\lambda^{+}\right)$. Hence, by Lemma $0.6, g r\left(\lambda^{+}\right)$is not simply connected. This shows that $a$ is in fact on edge. Now we wish to show that $\lambda^{+}=$ $\lambda_{a}$. Assume $\lambda^{+}=n \cdot \lambda_{a}$ and $n>1$. Applying Lemma 1.8 again, we find that then the $a$-string through $\lambda^{+}$contains at least the elements $\lambda^{+}, \lambda^{+}-a, \lambda^{+}-2 a$. But, since $\lambda^{+}\left(H_{b}\right)=0$ for $b \neq a$, choosing $b$ such that $(a, b)<0$ (which we can do since $R$ is simple and $\operatorname{rank}(R)>1)$, we find that $\left(\lambda^{+}-a\right)\left(H_{b}\right)>0$. Hence, by Lemma 1.8 again, both $\left(\lambda^{+}-a\right)-b$ and $\left(\lambda^{+}-a\right)-a$ are in $W t\left(\lambda^{+}\right)$and hence $\lambda^{+}-a$ is a branch point of $g r\left(\lambda^{+}\right)$. Hence $g r\left(\lambda^{+}\right)$, by Lemma 0.6, is not simply connected.

Proposition 1.13. Suppose $\pi=\pi\left(\lambda^{+}\right)$is s.c. Then the set of nonzero weights of $\pi$ is equal to the Weyl group orbit of $\lambda^{+}$. 0 may or may not be a weight.

Proof. We had seen (Proposition 1.5) that $W t^{<}\left(\lambda^{+}\right)=W \cdot \lambda^{+}$. So the proposition is equivalent to the statement that any nonextremal weight in $W t\left(\lambda^{+}\right)$is zero. So suppose $\lambda \in W t\left(\lambda^{+}\right), \lambda \notin W t^{<}\left(\lambda^{+}\right)$. Then for some fixed $a \in \Sigma^{0}$, the $a$-string through $\lambda$ contains the string in $\operatorname{gr}\left(\lambda^{+}\right), \lambda-a, \lambda, \lambda+a$. We wish to show $\lambda=0$. Now $\lambda=0$ iff $W_{\lambda}$, the stabilizer of $\lambda$ in $W$ equals $W$. The last statement, again, is equivalent to $w_{b}(\lambda)=\lambda$ for all $b \in \Sigma^{0}$. Hence the proposition boils down to proving that $\lambda \notin W t^{<}\left(\lambda^{+}\right)$implies $w_{b}(\lambda)=\lambda$ for $b \in \Sigma^{0}$. (Obviously this holds only for s.c. $\pi$.) First we show that $\lambda \notin$ $W t^{<}\left(\lambda^{+}\right)$implies $w_{a}(\lambda)=\lambda$. If not, then, choosing $b \in \Sigma^{0},(a, b)<0$, 
we see that at least one of $\lambda\left(H_{b}\right)$ or $\left(w_{a}(\lambda)\left(H_{b}\right)\right)$ is nonzero. Hence through at least one of $\lambda, w_{a}(\lambda)$ passes a nontrivial $b$-string. But since $\lambda$, and hence also $w_{a}(\lambda)$, are not $a$-extremal, since $w_{a}(a)=-a$, this implies that either $\lambda$ or $w_{a}(\lambda)$ (and hence both) is a branch point of $\operatorname{gr}\left(\lambda^{+}\right)$. Clearly for $b \neq a$, there can be no nontrivial $b$ string passing through $\lambda$. Hence $w_{b}(\lambda)=\lambda$.

CoROLLARY 1.14. If $\pi$ is s.c. then for all $a \in \Sigma^{0}$, all a-strings are of length 2 or $3 . \quad 0$ is a weight iff there exists (exactly) one achain of length 3.

Proof. This follows immediately from the fact that all nonzero weights must be extremal.

Proposition 1.12 reduces the complete identification for each simple algebra to a small number of cases. We now complete this identification. We state for future reference the following obvious

PROPOSITION 1.15. $\pi$ is s.c. iff $\pi^{2}$, the contragradient representation is s.c.

The proposition follows from the fact that the weight systems of $\pi$ and $\pi^{2}$ are each other's negatives.

Now we investigate the only candidates for s.c. representations, namely the ones of the form $\pi=\pi\left(\lambda^{+}\right)$, and $\lambda^{+}=\lambda_{a}$ with $a$ on edge. We have

LEMMA 1.16. Let $a_{1}, \cdots, a_{r}, r \leqq p=\operatorname{rank}(R)$ be an initial segment of nodes of the Dynkin diagram of $R$. Suppose the $a_{i}$ are all of the same length, i.e., the initial segment is as follows:

$$
0-0 \cdots-0 \cdots
$$

Then there exists a unique descending chain of length $r+1$ in $\operatorname{gr}\left(\lambda^{+}\right)$starting with $\lambda^{+}=\lambda_{a_{1}}$. The ith member of this chain is given by $w t_{i}=\lambda_{a}-\sum_{j=1}^{i-1} a_{j}$.

Proof. We prove this proposition by induction. For $i=1$ there is nothing to prove. So assume there to be a unique descending sequence $w t_{1}=\lambda_{a_{1}}, w t_{2}=\lambda_{a_{2}}, \cdots, w t_{i}=\lambda_{a_{1}}-\sum_{j=1}^{i-1} a_{j}$. We are interested in finding those $b \in \Sigma^{0}$ for which $w t_{i}\left(H_{b}\right)>0$ since those are exactly the ones for which a maximal descending $b$-chain begins with $w t_{i}$. We compute, setting $H_{i}=H_{a_{i}}$. 


$$
\left\{\begin{array}{l}
w t_{i}\left(H_{a_{1}}\right)=\lambda_{a_{1}}\left(H_{a_{1}}\right)-a_{1}\left(H_{a_{1}}\right)-a_{2}\left(H_{a_{1}}\right)=1-2+1=0 \\
w t_{i}\left(H_{j}\right)=-\left(a_{j-1}+a_{j}+a_{j+1}\right)\left(H_{j}\right)=0 \text { for } 1<j<i-1 \\
w t_{i}\left(H_{i-1}\right)=-\left(a_{i-2}-a_{i-1}\right)\left(H_{i-1}\right)=-1 \text { for } j=i-1 \\
w t_{i}\left(H_{i}\right)=-a_{i-1}\left(H_{i}\right)=1 \\
w t_{i}\left(H_{j}\right)=-\sum_{v=1}^{i-1} a_{v}\left(H_{j}\right)=0 \text { for } j>i .
\end{array}\right.
$$

(1.17) says that for precisely one $a \in \Sigma^{0}$, namely $a=a_{i}$ is $w t_{i}\left(H_{a_{i}}\right)>$ 0 . Hence an $a_{i}$-string descends from $w t_{i}$ and only such a string. This proves the lemma.

CoROLlary 1.18. Let $R=A_{p}$. Then there exactly two, mutually contragradient, representations with simply connected weight diagram. They are the two fundamental representations corresponding to the two roots on edge in the Dynkin diagram. They are both of dimension $p+1$. Their weight system constitutes a single orbit under $W$, each weight being of the form $\lambda_{a_{1}}-\sum_{i}^{m} a_{i}, m \leqq p .0$ is not a weight.

Proof. Let $\lambda^{+}=\lambda_{a}, a$ one of the two nodes 'on edge'. The previous lemma proves that there exists a unique descending chain of length $p+1$, in $g r\left(\lambda^{+}\right)$. A direct computation as in (1.17) shows that there is no chain of length $p+2$. The fact that all $a$-chains occurring in this unique descending chain are of length 2 implies, without direct computation, that 0 doesn't occur, since all $a$-chains containing an element $\lambda$ orthogonal to $a$ must be of odd length. This observation together with Proposition 1.13 implies that $W t\left(\lambda^{+}\right)$ constitutes a single Weyl group orbit.

Now it is immediately verified that $W t\left(\lambda^{+}\right) \neq-W t\left(\lambda^{+}\right)$. Hence $\pi\left(\lambda^{+}\right)$is not self-contragradient. Hence $\pi\left(\lambda_{a_{1}}\right)$ and $\pi\left(\lambda_{a_{p}}\right)$ are mutually contragradient since they are the only s.c. representations.

Proposition 1.19. Suppose $R$ is a simple Lie algebra whose Dynkin diagram has a branch point. Then there are no s.c. representations of $R$.

Proof. If an s.c. representation exists we have seen (Proposition 1.12) that it must be of the form $\lambda^{+}=\lambda_{a}$, with a on edge. The Dynkin diagram possesses a connected subgraph of the following sort:

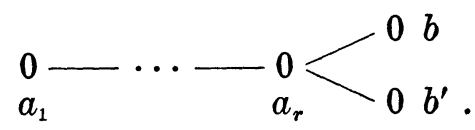


Now consider the naturally imbedded simple Lie algebras with Dynkin diagrams

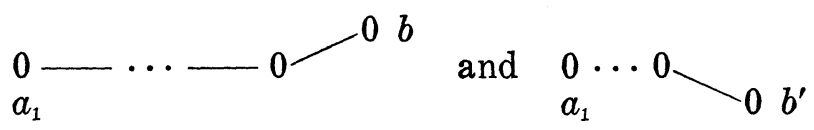

respectively. Reducing $\pi$ restricted to each of these algebras and applying Lemma 1.16 to each, we immediately obtain the existence of two initial chains in $g r\left(\lambda^{+}\right)$,

$$
\begin{array}{ll}
\lambda^{+}, \cdots, \lambda^{+}-\sum^{r} a_{j}, & \lambda^{+}-\sum^{r} a_{j}-b \\
\lambda^{+}, \cdots, \lambda^{+}-\sum^{r} a_{j}, & \lambda^{+}-\sum^{r} a_{j}-b^{\prime} .
\end{array}
$$

So there exists a branch point in $\operatorname{gr}\left(\lambda^{+}\right)$, namely $\lambda^{+}-\sum_{j}^{r} a_{j}$, and $g r\left(\lambda^{+}\right)$is not s.c.

Proposition 1.19 immediately yields the

Proposition 1.22. $D_{p}$ with $p>3, E_{8}, E_{7}$, and $E_{8}$ have no s.c. representations.

EXAMPLE 1.23. Consider the first fundamental representation of $D_{3}$, the Lie algebra of $S O(6)$. Its graph $g r\left(\lambda^{+}\right)$is as follows:

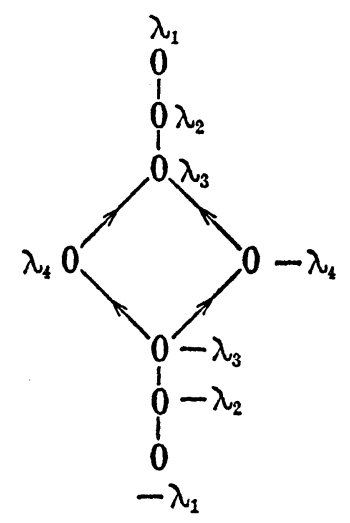

with the $\lambda_{i}$ 's being the usual dual coordinates in $K^{8}$ with respect to which the weight system consists of $\left[ \pm \lambda_{i}\right]_{i=1, \ldots, 4}$.

Having analyzed all algebras whose roots are all of one length, namely $A_{p}, D_{p}, E_{i}$, we still need to analyze Lie algebras with different sizes of roots, i.e., $B_{p}, C_{p}, G_{2}$ and $F_{4}$. We start with $C_{2}=B_{2}$.

Proposition 1.24. The two fundamental representations of $C_{2}$ given by (1.25) are s.c. They are of dimensions 4 and 5, and are the standard representations defining the symplectic algebra of 
dimension 4 and special orthogonal algebra of dimension 5, respectively. Their weight diagrams are as in (1.26) and (1.27) respectively, with respect to the enumeration of simple roots given in (1.25).
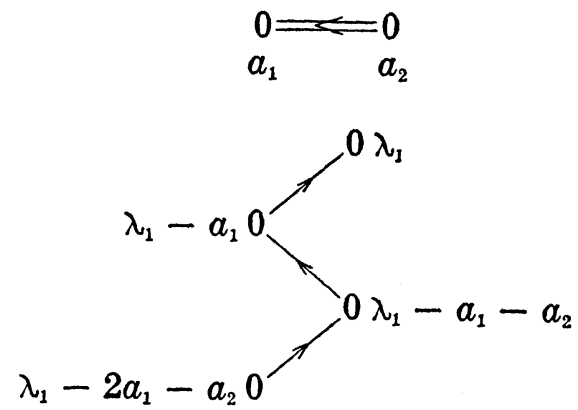

(1.27)

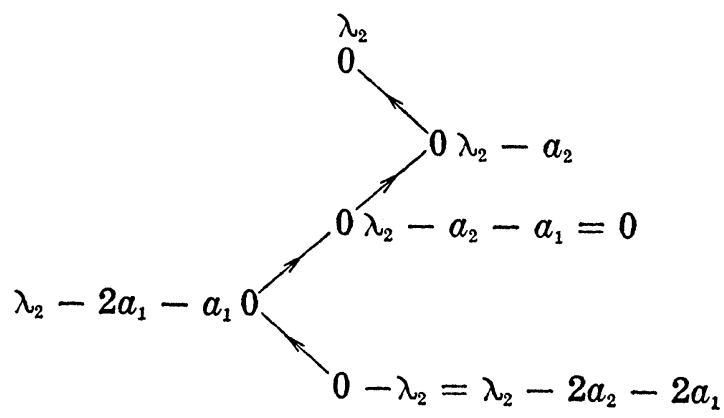

Proof. It is elementary to verify by direct computation that in each case there is a unique descending chain of lengths 4 and 5 respectively, determined by the sequence of simple roots for which $\lambda_{i}\left(H_{a_{i+1}}\right)>0$.

To analyze $C_{p}, B_{p}(p>2)$ and $F_{4}$, we first analyze initial segments of the form

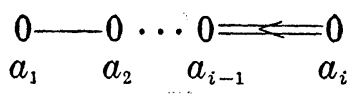

and

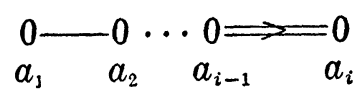

LEMma 1.29. For simple algebras having an initial segment as in (1.28a) and (1.28b) with $i>2$, the first fundamental representation of $R, \pi\left(\lambda^{+}\right)$with $\lambda^{+}=\lambda_{1}$ has an initial descending chain of the form (a) and (b) respectively (and letting $a_{0}=0$ ):

(a) $w t_{k}=\lambda_{1}-\sum_{j=1}^{k+1} a_{j-1}, k=0, \cdots, i$ $w t_{k+i}=w t_{i}-\sum_{j=1}^{k} a_{i-j}, k=1, \cdots, i-1$

(b) $w t_{k}=\lambda_{1}-\sum_{j=1}^{k+1} a_{j-1}, k=0, \cdots, i$ $w t_{k+i}=w t_{i}-\sum_{j=0}^{k} a_{i-j}, k=0, \cdots, i-1$. 
Furthermore, (a) and (b) are the unique descending chains of length $\leqq 2 i$ starting with $\lambda_{1}$ in case (1.28a) respectively, (1.28b), are the Dynkin diagram of $R$ (not just an initial segment). The proof is a straightforward computation.

CoROLLARY 1.30. $F_{4}$ has no s.c. representations.

Proof. The Dynkin diagram of $F_{4}$ is as follows:

$$
\begin{array}{cccc}
0-0 & \Longrightarrow & 0 & 0 \\
a_{1} & a_{2} & a_{3} & a_{4}
\end{array} \text {. }
$$

We apply the previous lemma first to the initial segment $a_{1}, a_{2}, a_{3}$ of this Dynkin diagram. Then, we obtain immediately that $\lambda_{1}-$ $\sum_{j=1}^{3} a_{j}$ is a downwards branch point of $g r\left(\lambda_{1}\right)$ since according to the lemma $\lambda_{1}-\sum_{1}^{3} a_{j}-a_{2}$ is in the weight system $W t\left(\lambda_{1}\right)$ and on the other hand $\left(\lambda_{1}-\sum_{1}^{3} a_{j}-a_{4}\left(H_{4}\right)\right)=1$, and hence $\lambda_{1}-\sum_{1}^{3} a_{j}-a_{4}$ is in $W t\left(\lambda_{1}\right)$. Hence $\operatorname{gr}\left(\lambda_{1}\right)$ is not simply connected.

Secondly we re-enumerate the Dynkin diagram (1.31) by $a_{i}^{\prime}=$ $a_{4-i+1}$ and apply the preceding lemma to the initial segment defined by $a_{j}^{\prime}, j=1,2,3$. Again we obtain that $\lambda_{1}-\sum_{1}^{3} a_{j}$ is a branch point.

COROLlaRY 1.32. Let $R=C_{p}, p>2$. Then there exists exactly one s.c. representation, namely the first fundamental representation with respect to the enumeration of simple roots

$$
\begin{array}{cccc}
0-0 & \cdots & 0=0 \\
a_{1} & a_{2} & a_{p-1} & a_{p}
\end{array}
$$

Furthermore the weight system of $\pi_{1}$ is given by

$$
\lambda_{1}, \cdots, \lambda_{1}-\sum_{j=1}^{p} a_{j}, \cdots, \lambda_{1}-\sum_{j=1}^{p} a_{j}-\sum_{j=p-1}^{1} a_{j} .
$$

Furthermore 0 does not occur as a weight and the weight system is one orbit under the Weyl group. All a-strings, $a \in \Sigma$, are of length 2 , and the jth weight is obtained by applying the jth element of the sequence of Weyl group elements $w_{j}$ to $\lambda^{+}=\lambda_{1}$ where the sequence of elements $w_{j}$ is as follows:

$$
w_{a_{1}}, \cdots, w_{a_{1}} \cdots w_{a_{p}}, w_{a_{1}} \cdots w_{a_{p}} w_{a_{p-1}}, \cdots, w_{a_{1}} \cdots w_{a_{p}} w_{a_{p-1}} \cdots w_{a_{1}} .
$$

Proof. The first assertion of the corollary, that $\pi\left(\lambda_{1}\right)$ is s.c., follows immediately from the last statement of the Lemma 1.29, in which the unique initial descending chain of length $2 p$ was given, since a direct computation shows that there is no chain of length $>$ $2 p$. The explicit computation of the lemma also shows that all $a$ - 
chains are of length 2, and hence 0 does not occur. Hence, by Proposition 1.13, $W t\left(\lambda_{1}\right)=W \cdot \lambda_{1}$. The explicit formula for a weight expressed as a product of reflections applied to $\lambda_{1}$ is a direct computation.

We consider the other candidates for an s.c. representation: $\lambda^{+}=\lambda_{a_{p}}$. We obtain the following two descending chains starting with $\lambda^{+}$(we assume $p>2$ ):

$$
\begin{aligned}
& \lambda^{+}, \lambda^{+}-a_{p}, \lambda^{+}-a_{p}-a_{p-1}, \lambda^{+}-a_{p}-2 a_{p-1} \\
& \lambda^{+}, \lambda^{+}-a_{p}, \lambda^{+}-a_{p}-a_{p-1}, \lambda^{+}-a_{p}-a_{p-1}-a_{p-2} .
\end{aligned}
$$

Hence $\lambda^{+}-a_{p}-a_{p-1}$ is a downward branch point and $\pi$ is not s.c.

CoRollary 1.34. Let $R=B_{p}$, with $p>2$ and Dynkin diagram given by

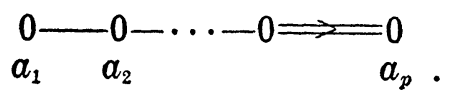

Then there exists exactly one s.c. representation, the one with fundamental weight $\lambda^{+}=\lambda_{a_{1}}$. Its weight system consists of the Weyl group orbit of $\lambda^{+}$and the 0 weight. All a-strings are of length 2 except one which is of length 3 . It is the $a_{p}$-string. The weight system is as in (1.35). It is of dimension $2 p+1$. The highest weight is the sum of the simple roots.

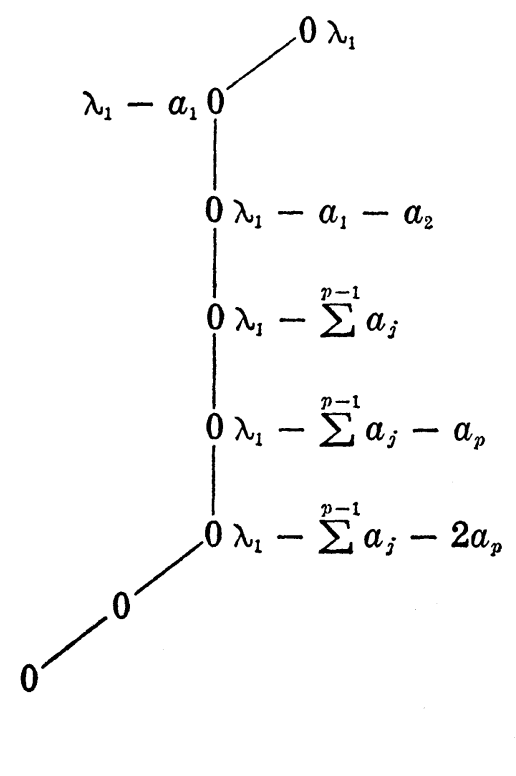


Proof. The statements about $\pi=\pi\left(\lambda_{1}\right)$ follow again from the computations of the lemma. To see that $\pi\left(\lambda_{1}\right)$ is the unique s.c. representation of $B_{p}, p>2$, one simply observes that for $\pi=\pi\left(\lambda_{p}\right)$ (the only other candidate) $\lambda^{+}-a_{p}-a_{p-1}$ is a downward branch point, and hence $\operatorname{gr}\left(\lambda_{p}\right)$ is not simply connected.

REMARK. The unique s.c. representations of $C_{p}$ and $B_{p}$ found in Corollaries 1.32 and 1.34 are of course the 'natural' representations which are used to define the 'symplectic' and 'orthogonal' algebras.

Proposition 1.36. If $R=G_{2}$ then there exists exactly one s.c. representation, namely the representation with fundamental weight $\lambda^{+}=\lambda_{a_{1}}$ for the enumeration

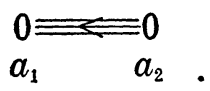

Its weight system is as follows:

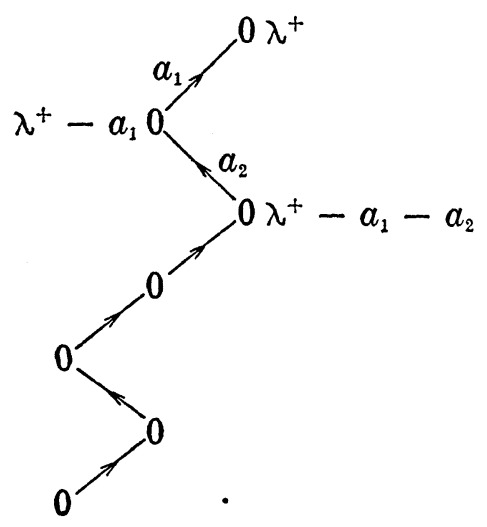

Its weight system consists of one (singular) orbit and the zero weight.

Proof. Again, using Lemma 1.8, we can readily compute the weight graph $g r\left(\lambda_{1}\right)$ as given in (1.37), from which the last assertion can be read off directly.

We have now determined the s.c. representations of all isomorphism types of split simple Lie algebras, and have verified the assertions of Theorem 1.7. We now have an elementary procedure for constructing an s.c. representation, by giving the matrix coefficients explicitly.

LEMMA 1.38. If $\pi$ is s.c. then all weights of $\pi$ occur with 
multiplicity one.

Proof. This is immediate from the standard facts outlined in the introduction.

REMARK. The converse of the lemma is false. See, for instance, the standard representation of $D_{p}, p>2$, which is not s.c., but whose weights all have multiplicity one.

The most remarkable property of s.c. representations is the following:

THEOREM 1.39. If $\pi$ is an s.c. representation then there exists $a$ basis in the representation space with respect to which $\pi_{a}$ is in Tits normal form, for all $a \in \Sigma^{0}$ simultaneously.

Proof. We fix a sequence of vectors $v_{1}^{1}, \cdots v_{r_{1}}^{1}, \cdots, v_{1}^{s}, \cdots, v_{r_{s}}^{s}$ having the following properties:

(i) for each $i$ between 1 and $s, v_{1}^{i}, \cdots, v_{r_{i}}^{i}$ is a basis in a nontrivial irreducible $t_{a_{j_{i}}}$-module, $V^{i}$ on which one of the R.S.A.'s acts by restriction of $\pi$, with respect to which that restriction, $\pi_{a_{j_{i}}}$ is in Tits normal form.

(ii) $v_{r_{i}}^{i}=c^{i} \cdot v_{1}^{i+1}$.

The fact that such a set exists immediately follows from the previous lemma and the existence of Tits normal forms in the irreducible representations $\pi_{a}$ of $\mathrm{sl}(2)$. We now construct a sequence of bases of $V, B_{1}, \cdots, B_{s}$ and a sequence of sequences of vectors $v_{j}^{i}$ such that $\pi_{a_{j_{i}}}$ will be in Tits normal form with respect to $B_{r}$ for $i \leqq r$. In particular all $\pi_{a}$ 's will be in Tits normal form with respect to $B_{8}$.

We choose $B_{1}$ to be the basis $v_{1}^{1}, \cdots, v_{r_{1}}^{1}, v_{2}^{2}, \cdots, v_{r_{2}}^{2}, \cdots, v_{2}^{s}, \cdots, v_{r_{s}}^{s}$. Obviously $\pi_{a_{j_{1}}}$ is in Tits normal form with respect to $B_{1}$. Having chosen $B_{i}$, we define $B_{i+1}$ to be $M_{i}\left(B_{i}\right)$, where $M_{i}$ is the following matrix:

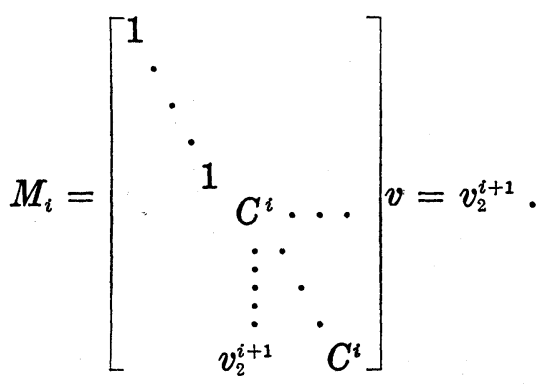


We also apply $M_{i}$ to the sequence $v_{2}^{i+1}, \cdots, v_{r_{s}}^{s}$, leaving the preceding $v$ 's the same. We define $v_{1}^{i+1}=v_{r_{i}}^{i}$, and $B_{i+1}$ to be the basis defined in the same way as $B_{1}$, but with respect to the modified set $v_{j}^{1}$. It is clear that under the inductive hypothesis, $\pi_{a_{j_{m}}}$ has Tits normal form with respect to $B_{i+1}$ for $m \leqq i+1$.

2. Geometry of the weight system: Weight polyhedron and weight lattices. The weight graphs $\operatorname{gr}\left(\lambda^{+}\right)$and $\operatorname{Gr}\left(\lambda^{+}\right)$are naturally imbedded in the rational space $\sqrt{{ }^{\prime}}$. We now study this geometric object and give a geometric characterization of the weight system $W t\left(\lambda^{+}\right)$. We know that $\Sigma^{0}$ is a $Q$-basis of $\sqrt{ }^{{ }^{0}}$, and that $\sqrt{ }^{{ }^{\prime}}$ is a $Q$ inner product space with respect to the Killing form (, ). By a rational polyhedron in a rational inner product space $\sqrt{ }$ we shall mean the convex closure of a finite number of points in $\sqrt{ }$. Note that the closure of a rational polyhedron in the corresponding real space is a polyhedron in the ordinary sense.

Now we introduce the following data: We define the 'representation lattice' of $\pi\left(\lambda^{+}\right)$to be

$$
L\left(\lambda^{+}\right)=\lambda^{+}+L\left(\Sigma^{0}\right)
$$

and the 'representation cone'

$$
L^{-}\left(\lambda^{+}\right)=\lambda^{+}-\left(L\left(\Sigma^{0}\right)\right)^{+} .
$$

So $L^{-}\left(\lambda^{+}\right)=\left\{\lambda^{+}-\sum_{\Sigma^{0}} n_{a} \cdot a /\left(n_{a}\right) \in N^{p}\right\}$. On $L^{-}\left(\lambda^{+}\right)$, and any subset of it, we define the 'height function' by the formula

(2.3) $h(\lambda)=\sum_{a \in \Sigma^{0}} n_{a}$ for the unique expression of $\lambda$ as $\lambda=\lambda^{+}-\sum_{20} n_{a} \cdot a$.

In the following we shall be making use of induction with respect to the $N$-valued function $h$.

The first observation we make is the easily verified

Lemma 2.4. $L\left(\lambda^{+}\right)$is preserved by the Weyl group.

Note that $L^{-}\left(\lambda^{+}\right)$is not preserved by $W$.

Next, we define the 'weight polyhedron' attached to $\pi$, or $\lambda^{+}$, as

(2.5) $C=C\left(\lambda^{+}\right)=$convex closure of $W \cdot \lambda^{+}$, the Weyl group orbit of $\lambda^{+}$.

We define $C^{>}$to be the set of extremal points of $C$, i.e. the set of points $c$ in $C$ which are only trivially convex combinations $c=\Sigma \mathrm{d}_{i} \cdot c_{i}$, with $d_{i} \in Q$ and $c_{i} \in C\left(d_{i}>=0, \Sigma d_{i}=1\right)$. We want to show that this set equals the set of extremal weights introduced in $\S 1$, establishing the first interesting relation between the geometry 
of $C$ and the representation theory of $\pi\left(\lambda^{+}\right)$.

LEMma 2.6. Let some finite group $W$ act linearly on an inner product space $V$, and act transitively on some finite set $S$. Then the set of extremal points of the convex closure of $S$ equals $S$.

Proof. Since $W$ is finite, we can assume the action of $W$ to be orthogonal with respect to the inner product. The transitivity of the action then assures that $S$ is contained in the unit sphere (renormalizing perhaps) of $V$. The lemma then follows from the fact that any line segment connecting two points on the unit sphere meets the sphere only in those two points.

We have immediately, for s.c. $\lambda^{+}$

Corollary 2.7. $C^{>}\left(\lambda^{+}\right)=W t^{<}\left(\lambda^{+}\right)=W \cdot \lambda^{+}$.

Proof. The second equality was established in $\S 1 . C^{>}\left(\lambda^{+}\right)=$ $W \cdot \lambda^{+}$by the definition of $C\left(\lambda^{+}\right)$, in view of the preceding lemma.

Next we have

LEMmA 2.8. $C\left(\lambda^{+}\right) \cap L\left(\lambda^{+}\right) \subseteq L^{-}\left(\lambda^{+}\right)$.

Proof. In fact we prove that any convex combination of weights of $\pi\left(\lambda^{+}\right)$lies in the negative affine cone $\lambda^{+}-\sum_{i=1}^{n} d_{i} \cdot a_{i}\left(d_{i}>=0\right)$, which will prove the claim. So let $v=\sum d_{i} \cdot \lambda_{i}$, some convex combination of weights. Expressing each $\lambda_{i}$ as

$$
\lambda_{i}=\lambda^{+}-\sum_{a \in \Sigma^{0}} d_{i, a} \cdot a,
$$

we obtain

$$
\begin{aligned}
v & =\sum_{i} d_{i}\left(\lambda^{+}-\sum_{a} d_{i, a} \cdot a\right) \quad\left(\text { since } \sum_{i} d_{i}=1\right) \\
& =\lambda^{+}-\sum_{a}\left(\sum_{i} d_{i} \cdot d_{i, a}\right) a
\end{aligned}
$$

and the coefficients of the $a$ 's in the last expression are all nonnegative.

The point of the lemma is that we can apply induction with respect to $h$ to $C\left(\lambda^{+}\right) \cap L\left(\lambda^{+}\right)$, and any of its subsets.

Next we have

LeMma 2.9. $C\left(\lambda^{+}\right) \cap L^{-}\left(\lambda^{+}\right)$is preserved by the Weyl group.

Proof. By the preceding lemma, $C\left(\lambda^{+}\right) \cap L^{-}\left(\lambda^{+}\right)=C\left(\lambda^{+}\right) \cap L\left(\lambda^{+}\right)$. 
Since each of these sets is preserved by the Weyl group (Lemma 2.4 ), so is their intersection.

We can now prove the geometric characterization of $W t\left(\lambda^{+}\right)$ which we seek.

THEOREM 2.10. $W t\left(\lambda^{+}\right)=C\left(\lambda^{+}\right) \cap L\left(\lambda^{+}\right)=\bigcup_{w \in W} w\left(C^{+} \cap L^{-}\left(\lambda^{+}\right)\right)$.

Proof. ( i ) $W t\left(\lambda^{+}\right) \subseteq C\left(\lambda^{+}\right) \cap L\left(\lambda^{+}\right)$. We already know $W t\left(\lambda^{+}\right) \subseteq$ $L^{-}\left(\lambda^{+}\right) \subseteq L\left(\lambda^{+}\right)$. For the second inclusion $W t\left(\lambda^{+}\right) \subseteq C\left(\lambda^{+}\right)$we use induction using our function $h(\lambda)$ (2.3). Starting with the trivial case $h(\lambda)=0$, i.e., $\lambda=\lambda^{+}$, we assume now that $\lambda$ be an $h$-minimal counterexample to $W t\left(\lambda^{+}\right) \subseteq C\left(\lambda^{+}\right)$. Then $\lambda$ can't be an extremal weight since those weights are already in $C\left(\lambda^{+}\right)^{>}=W t^{<}\left(\lambda^{+}\right)=W \cdot \lambda^{+}$. So we can choose $a \in \Sigma^{0}$ such that $\lambda$ is an interior member of an $a$-chain, i.e. " $\lambda-a, \lambda, \lambda+a$ are all in $W t\left(\lambda^{+}\right)$. Letting $\lambda^{\prime}, \lambda^{\prime \prime}$ be the highest and lowest member of this $a$-string $\left(\lambda^{\prime}, \lambda^{\prime \prime} \neq \lambda\right)$ we see that $\lambda^{\prime} \in C\left(\lambda^{+}\right)$since $h\left(\lambda^{\prime}\right)<h(\lambda)$. But $\lambda^{\prime \prime}=w_{a}\left(\lambda^{\prime}\right)$ and hence $\lambda^{\prime \prime} \in C\left(\lambda^{+}\right)$. Hence $\lambda$, lying on the line between those two must be in $C\left(\lambda^{+}\right)$.

(ii) $C\left(\lambda^{+}\right) \cap L\left(\lambda^{+}\right) \subseteq \mathbf{U}_{w \in W} w\left(\underline{C}^{+} \cap L^{-}\left(\lambda^{+}\right)\right)$. To show this it obviously suffices to show that

$$
C\left(\lambda^{+}\right) \cap L\left(\lambda^{+}\right) \cap \underline{C}^{+}=C\left(\lambda^{+}\right) \cap L^{-}\left(\lambda^{+}\right) \cap \underline{C}^{+} \subseteq C^{+} \cap L^{-}\left(\lambda^{+}\right),
$$

by the transitivity of the Weyl group and the invariance of $C\left(\lambda^{+}\right) \cap L^{-}\left(\lambda^{+}\right)$under the Weyl group (Lemma 2.9). But (2.11) is a triviality.

It remains to show, and this is the crux of the matter

(iii) $\mathrm{U}_{w \in W} w\left(C^{+} \cap L^{-}\left(\lambda^{+}\right)\right) \subseteq W t\left(\lambda^{+}\right)$. Once again, by Weyl group invariance of both sides of this relation, it suffices to show that

$$
\underline{C}^{+} \cap L^{-}\left(\lambda^{+}\right) \subseteq W t\left(\lambda^{+}\right) \cap \underline{C}^{+}
$$

First we have

LEMma 2.13. If for some $a \in \Sigma^{0}, n \in N, \lambda=\lambda^{+}-n \cdot a \in \underline{C}^{+}$then $\lambda \in W t\left(\lambda^{+}\right)$.

Proof. The lemma immediately follows from the fact that for the lowest member of the $a$-chain through $\lambda^{+}$, we have $\lambda\left(H_{a}\right) \leqq 0$, and equality holds only if $\lambda^{+}\left(H_{a}\right)=0$. Hence either $\lambda=\lambda^{+}$, or $\lambda\left(H_{a}\right)<0$ and hence $\lambda \notin \underline{C}^{+}$.

Next, we have

LEMMA 2.14. Let $T \subseteq \Sigma^{0}$ be an orthogonal family of simple roots. 
Then $\left\{w_{a} \mid a \in T\right\}$ is a commuting family of reflections. Hence $w(T)=$ $\Pi_{a \in T} w_{a}$ is of order 2. Furthermore, $w(T)(\lambda)=\lambda-\sum_{a \in T} \lambda\left(H_{a}\right) \cdot a$.

Proof. The lemma is immediate from standard theory.

Now we prove (2.12). Suppose it is false. Pick some $\lambda \in \underline{C}^{+} \cap$ $L^{-}\left(\lambda^{+}\right), \lambda \notin W t\left(\lambda^{+}\right)$, for which $h(\lambda)$ is minimal (minimal among all counterexamples in $\underline{C}^{+} \cap L^{-}\left(\lambda^{+}\right)$). Now by the Lemma 2.13 there exists a set $S \subseteq \Sigma^{0}$ containing at least two elements such that we can write

$$
\lambda=\lambda^{+}-\sum_{S} n_{a} \cdot a
$$

with $n_{a}>0$ for all $a \in S$. Hence, by standard theory, we can pick $a \in S$ such that for all $b \in \Sigma^{0}, a\left(H_{b}\right)=-1$ or $a\left(H_{b}\right)=0$. We fix such on $a$. We now examine the element $\lambda+a$. Since $h(\lambda+a)<h(\lambda)$, we conclude, by $h$-minimality, that $\lambda+a \notin \underline{C}^{+} \cap L^{-}\left(\lambda^{+}\right)$. But, since $a \in S$, clearly $\lambda+a \in L^{-}\left(\lambda^{+}\right)$. Hence $\lambda+a \notin \underline{C}^{+}$. Now define $T_{a} \subseteq \Sigma^{0}$ to be the set of simple roots for which $(\lambda+a)\left(H_{b}\right)<0$. For all $b \in T_{a},(a, b) \neq 0$, and hence $T_{a}$ is an orthogonal family of simple roots. $T_{a}$ is nonempty since $\lambda+a \notin \underline{C}^{+}$. We show that for $b \in T_{a}$, $\lambda\left(H_{b}\right)=0$. This follows immediately from $(\lambda+a)\left(H_{b}\right)=\lambda\left(H_{b}\right)+$ $a\left(H_{b}\right)=\lambda\left(H_{b}\right)-1$, since $\lambda \in \underline{C}^{+}$. This implies that $b \in S$ : writing

$$
0=\lambda\left(H_{b}\right)=\left(\lambda^{+}-\sum_{c \in S \backslash\{b\}} n_{c} \cdot c\right)\left(H_{b}\right)-n_{b} b\left(H_{b}\right),
$$

and $a\left(H_{b}\right)=-1$ implies that $n_{b}>=1 / 2$. But $n_{b}$ is an integer, hence $>=1$. We conclude that $T_{a} \subseteq S$. We now apply $w\left(T_{a}\right)$, as in Lemma 2.14 , to $\lambda+a$ : by the lemma we obtain $w\left(T_{a}\right)(\lambda+a) \in \underline{C}^{+}$. Furthermore, again by the lemma we have

$$
w\left(T_{a}\right)(\lambda+a)=\lambda+a-\sum_{b \in T_{a}}(\lambda+a)\left(H_{b}\right) \cdot b .
$$

But we just saw that $(\lambda+a)\left(H_{b}\right)=-1$. So (2.16) can be rewritten as

$$
w\left(T_{a}\right)(\lambda+a)=\lambda+a+\sum_{T_{a}} b .
$$

Since $a \in S$, and $T_{a} \leqq S, \quad(2.17)$ implies immediately that $w\left(T_{a}\right)(\lambda+a) \in L^{-}\left(\lambda^{+}\right)$. Since obviously $h\left(w\left(T_{a}\right)(\lambda+a)\right)<h(\lambda)$, the $h$-minimality of $\lambda$ as a counterexample to being in $W t\left(\lambda^{+}\right)$implies that $w\left(T_{a}\right)(\lambda+a) \in W t\left(\lambda^{+}\right)$. By Weyl group invariance of $W t\left(\lambda^{+}\right)$ this implies that $\lambda+a \in W t\left(\lambda^{+}\right)$. But

$$
(\lambda+a)\left(H_{a}\right)=\lambda\left(H_{a}\right)+a\left(H_{a}\right)>=2 .
$$

Hence $(\lambda+a)-a,(\lambda+a)-2 \cdot a$ must be weights. In particular $\lambda$ 
is a weight, and we have derived a contradiction to $\lambda$ being a counterexample to $\underline{C}^{+} \cap L^{-}\left(\lambda^{+}\right) \subseteq W t\left(\lambda^{+}\right)$.

This proves the theorem.

\section{REFERENCES}

1. N. Jacobsen, Lie Algebras, Interscience, 1962.

2. Jacques Tits, Sur les Constantes de Structure et le Theoreme d'Existence des Algebres de Lie Semi-simples, Publ. Math. I.H.E.S., 31 (1966), 21-58.

Received September 15, 1979. This paper is essentially a portion of the author's Berkeley $\mathrm{Ph}$. D. thesis. The author expresses his gratitude to his thesis advisor, Professor Calvin Moore for his encouragement and support. $\mathrm{He}$ also thanks Professor Gerhard Hochschild for many useful conversations.

\section{IHES}

BURES-SUR-YVETTE

France 



\section{PACIFIC JOURNAL OF MATHEMATICS}

\section{EDITORS}

DONALD BABBITT (Managing Editor)

J. DUGUNDJI

University of California

Los Angeles, California 90024

Hugo Rossi

University of Utah

Salt Lake City, UT 84112

C. C. Moore and Arthur Agus

Department of Mathematics

University of Southern California

Los Angeles, California 90007

R. FinN and J. Milgram

Stanford University

Stanford, California 94305

University of California

Berkeley, CA 94720

\section{ASSOCIATE EDITORS}
R. ARNES
E. F. BeCKenBaCH
B. H. NEUMANN
F. WoLF
K. YoshidA

\section{SUPPORTING INSTITUTIONS}

UNIVERSITY OF ARIZONA

UNIVERSITY OF BRITISH COLUMBIA

CALIFORNIA INSTITUTE OF TECHNOLOGY

UNIVERSITY OF CALIFORNIA

MONTANA STATE UNIVERSITY

UNIVERSITY OF NEVADA, RENO

NEW MEXICO STATE UNIVERSITY

OREGON STATE UNIVERSITY
UNIVERSITY OF OREGON

UNIVERSITY OF SOUTHERN CALIFORNIA

STANFORD UNIVERSITY

UNIVERSITY OF HAWAII

UNIVERSITY OF TOKYO

UNIVERSITY OF UTAH

WASHINGTON STATE UNIVERSITY

UNIVERSITY OF WASHINGTON 


\section{Pacific Journal of Mathematics}

\section{Vol. 101, No. $1 \quad$ November, 1982}

Natália Bebiano, On the evaluation of permanents $\ldots \ldots \ldots \ldots \ldots \ldots \ldots \ldots$

David Borwein and Bruce Brigham Watson, Tauberian theorems between

the logarithmic and Abel-type summability methods $\ldots \ldots \ldots \ldots \ldots \ldots 11$

Leo George Chouinard, II, Hermite semigroup rings $\ldots \ldots \ldots \ldots \ldots \ldots \ldots$

Kun-Jen Chung, Remarks on nonlinear contractions $\ldots \ldots \ldots \ldots \ldots \ldots . \ldots 4$

Lawrence Jay Corwin, Representations of division algebras over local

fields. II ......................................... 49

Mahlon M. Day, Left thick to left lumpy—a guided tour $\ldots \ldots \ldots \ldots \ldots 71$

M. Edelstein and Mo Tak Kiang, On ultimately nonexpansive

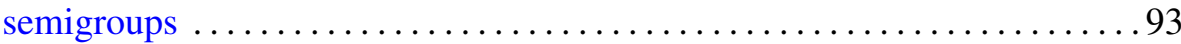

Mary Rodriguez Embry, Semigroups of quasinormal operators . ........ 103

William Goldman and Morris William Hirsch, Polynomial forms on

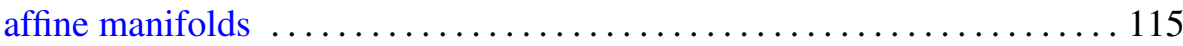

S. Janakiraman and T. Soundararajan, Totally bounded group topologies

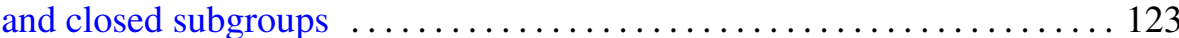

John Rowlay Martin, Lex Gerard Oversteegen and Edward D.

Tymchatyn, Fixed point set of products and cones $\ldots \ldots \ldots \ldots \ldots \ldots 133$

Jan van Mill, A homogeneous Eberlein compact space which is not metrizable ........................................ 141

Steven Paul Plotnick, Embedding homology 3-spheres in $S^{5} \ldots \ldots \ldots \ldots 147$

Norbert Riedel, Classification of the $C^{*}$-algebras associated with minimal rotations

Benedict Seifert, Combinatorial and geometric properties of weight systems of irreducible finite-dimensional representations of simple split Lie algebras over fields of 0 characteristic

James E. Simpson, Dilations on locally convex spaces

Paolo M. Soardi, Schauder bases and fixed points of nonexpansive mappings

Yoshio Tanaka, Point-countable $k$-systems and products of $k$-spaces

Fausto A. Toranzos, The points of local nonconvexity of starshaped sets . . . 209

Lorenzo Traldi, The determinantal ideals of link modules. I . . . . . . . . 215

P. C. Trombi, Invariant harmonic analysis on split rank one groups with applications

Shinji Yamashita, Nonnormal Blaschke quotients 